\title{
Scale Invariance and Self-averaging in disordered systems.
}

\author{
Giorgio Parisi ${ }^{a}$, Marco Picco $^{b}$ and Nicolas Sourlas ${ }^{c}$ \\ ${ }^{a}$ Dipartimento di Fisica, INFM, SMC and INFN, \\ Università di Roma La Sapienza, \\ P. A. Moro 2, 00185 Rome, Italy \\ ${ }^{b}$ Laboratoire de Physique Théorique et Hautes Energies ${ }^{1}$, \\ Boîte 126, Tour 16, $1^{\text {er }}$ étage, \\ 4 place Jussieu, F-75252 Paris CEDEX 05, France \\ ${ }^{c}$ Laboratoire de Physique Théorique de l'Ecole Normale Supérieure ${ }^{2}$, \\ 24 rue Lhomond, 75231 Paris CEDEX 05, France
}

\begin{abstract}
In a previous paper [1] we found that in the random field Ising model at zero temperature in three dimensions the correlation length is not self-averaging near the critical point and that the violation of self-averaging is maximal. This is due to the formation of bound states in the underlying field theory. We present a similar study for the case of disordered Potts and Ising ferromagnets in two dimensions near the critical temperature. In the random Potts model the correlation length is not self-averaging near the critical temperature but the violation of self-averaging is weaker than in the random field case. In the random Ising model we find still weaker violations of self-averaging and we cannot rule out the possibility of the restoration of self-averaging in the infinite volume limit.
\end{abstract}

PACS numbers: $05.10,05.20,75.10,75.40$

\footnotetext{
${ }^{1}$ Unité Mixte de Recherche CNRS UMR 7589, associée à l'Université Pierre et Marie Curie, PARIS VI et à l'Université Denis Diderot, PARIS VII.

${ }^{2}$ Unité Mixte de Recherche du CNRS et de l'Ecole Normale Supérieure, associée à l'Université Pierre et Marie Curie, PARIS VI.
} 
One of the important issues in the physics of disordered systems is the understanding of which physical quantities are self averaging, i.e. for which observables the sample to sample fluctuations vanish in the thermodynamic limit [4, 3].

In a recent work [1] we studied the behavior of the correlation length (CL) of the random field Ising model (RFIM) in three dimensions at zero temperature. We found surprisingly that the CL is not self-averaging and that the violation of self-averaging is maximal in the vicinity of the critical point. This is due to the formation of bound states in the underlying replica field theory. The interaction among replicas is attractive and therefore it has the capability of forming bound states. In the formation of bound states there is competition between the strength of the attractive forces and the size of the available phase space. We have argued that in two dimensions phase space is small and even a small attraction should win.

In this paper we address the question of the existence of bound states and their consequence, the non self-averaging of the CL, in the case of two disordered ferromagnetic spin models in two dimensions: the $q=3$ states Potts model and the Ising model. It is known that, in agreement with the Harris criterion [2], the disorder is relevant for the $q=3$ states Potts model, i.e. the renormalization group flows to a stable fixed point, which is different from the fixed point of the pure system [5, 6]. For the Ising model the disorder is marginally irrelevant and the renormalization group flows back to the fixed point of the pure system with logarithmic corrections [7.

In the case of the Potts model we find very similar behavior as in the RFIM. A scaling analysis shows that, as in the RFIM, the violation of self-averaging of the CL, although weaker than in the RFIM, persists for arbitrarily large systems in the vicinity of the critical point.

In the case of the Ising model, the violation of self-averaging is still weaker. The scaling analysis seems to work but we cannot exclude a very slow restoration of self-averaging in the thermodynamic limit (see later).

We conclude that the non self-averaging of the CL is not a singularity of the RFIM at zero temperature, but a more general phenomenon in the physics of disordered systems. The correlation length plays a crucial role in the scaling theories of phase transitions. It is not clear yet what are the consequences of the absence of self-averaging of the CL for those scaling theories in disordered systems.

In both the Potts and the Ising models we consider the case of square lattices of size $L^{2}$ where $L=200,350$, and 500, with nearest neighbor interactions. The ferromagnetic couplings $J$ are independent random variables which can take the two values $J=J_{1}$ and $J=J_{2}$ with equal probability. It is well known that these models are self-dual and that the critical inverse temperature $\beta=\beta_{c}$ is given by $[\underline{8}$ :

$$
\left(\exp \beta_{c} J_{1}-1\right)\left(\exp \beta_{c} J_{2}-1\right)=q .
$$

The knowledge of $\beta_{c}$ greatly simplifies the scaling analysis. It is also known that cluster algorithms [9] are very effective for these models. We simulated 1000 samples for $L=500$, 2000 for $L=350$ and 10000 for $L=200$. As in the case of the RFIM[1, we measured the correlation length by studying the dependence on the boundary conditions. More precisely in the Ising case (the generalization to the Potts case is obvious) we set all the spins equal to one on the line $x=0$ and choose free boundary conditions at the other end of the lattice $x=L$. We impose periodic boundary conditions on the perpendicular direction. After thermalisation, for every sample $s$ we measure the magnetization $m_{s}(x)=1 / L \sum_{y}<\sigma(x, y)>$ and study its dependence on $x$ and on the inverse temperature $\beta$. For the Potts model we studied extensively the case $J_{1} / J_{2}=10[1]$ and $\beta / \beta_{c}=.95, .96, .97, .98, .99, .995$ and 1 . We verify thermalisation in the following ways. For every realization of the couplings, we simulate two copies with different initial conditions. In one copy all spins are set to +1 and in the other they are chosen at random (except on the line $x=1$, where they are set to +1 ). Thermalisation time $t_{t h}$ 


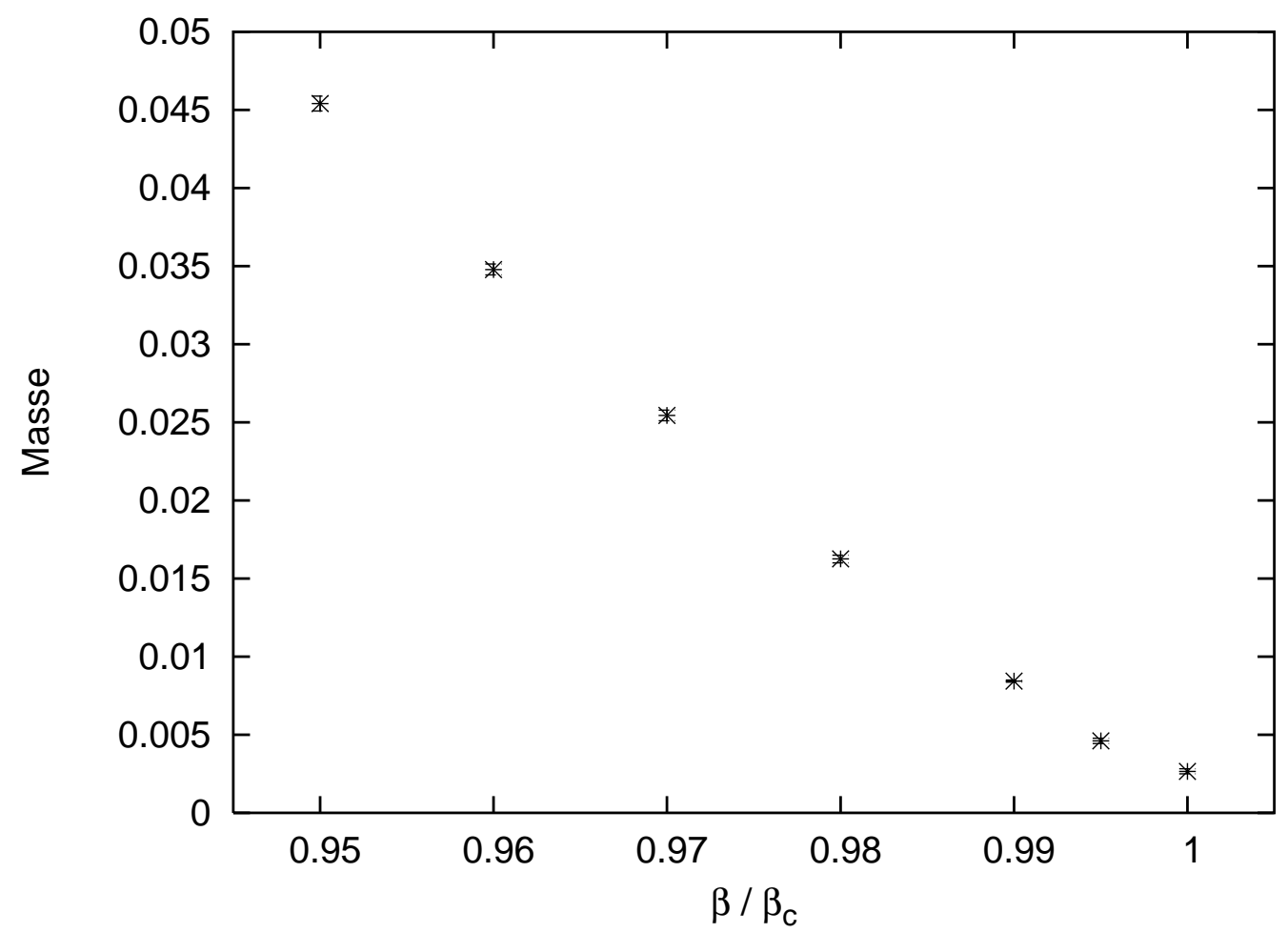

Figure 1: Mass as a function of $\beta<\beta_{c}$ for the $q=3$ Potts model and $L=500$.

must be long enough in order for the magnetization $m_{s}(x)$ of the 2 copies to be indistinguishable. The local magnetizations $\langle\sigma(x, y)\rangle$ are the time averages of the local spins during the measuring time $t_{m}$ We measure the autocorrelation time $\tau$ and require both the thermalisation time $t_{t h}$ and the measuring time $t_{m}$ to be much larger than $\tau$. We also verify that there are no time drifts in the measurements, i.e. averages over the first half of $t_{m}$ do not differ from measurements over the second half of $t_{m}$. Even at $\beta=\beta_{c}$ and for the volumes we simulated, $t_{t h}$ is finite and reasonably small because of the cluster algorithm. For $q=3$, we measured $\tau_{L=200} \simeq 200, \tau_{L=350} \simeq 400, \tau_{L=500} \simeq 800$ and we choose $t_{t h}=10 \tau, t_{m}=20 \tau$.

To reduce errors, we found convenient to use a variance reduction technique: we measured the probability $p(x, y)$ of the spin $\sigma(x, y)$ to be on the same cluster with the spins at the boundary $x=0$. Obviously $\langle\sigma(x, y)\rangle=2 p(x, y)-1$. In this way we eliminate statistical fluctuations due to flips of large clusters.

In order to study sample to sample fluctuations, we measured, as in the case of the RFIM[1], $m(x)=\overline{m_{s}(x)}$ and $m_{(2)}(x)=\overline{m_{s}(x)^{2}}$, where, as usually, the bar denotes average over the random coupling samples. As it was mentioned earlier, for every sample we simulated two copies, differing only at the initial conditions. In order to reduce errors, we measure the probability $p_{2}(x, y)$ that in both of the two copies we simulated, the spin at the site $(x, y)$ belongs to the cluster connected to the boundary at $x=0$. Obviously $<\sigma_{1}(x, y)><\sigma_{2}(x, y)>=$ $2 p_{2}(x, y)-1$ where $\sigma_{1}$ and $\sigma_{2}$ are the spins of the two copies. With these definitions, $m_{(2)}(x)=$ $1 / L \sum_{y} 2 \overline{p_{2}(x, y)}-1$.

For $\beta<\beta_{c}$, we expect $m(x)$ and $m_{(2)}(x)$ to be exponentially decreasing functions of $x$. We fitted $m(x)$ with the formula

$$
m(x)=\frac{\exp (-\mu x)}{x^{\alpha}}\left(a+\frac{b}{x}\right) .
$$

We found that this formula fits very well the data for all values of $x$ except near the boundaries $x \sim 0$ and $x \sim L$ and this for all values of $\beta$ we studied. The mass $\mu$ (or inverse correlation 


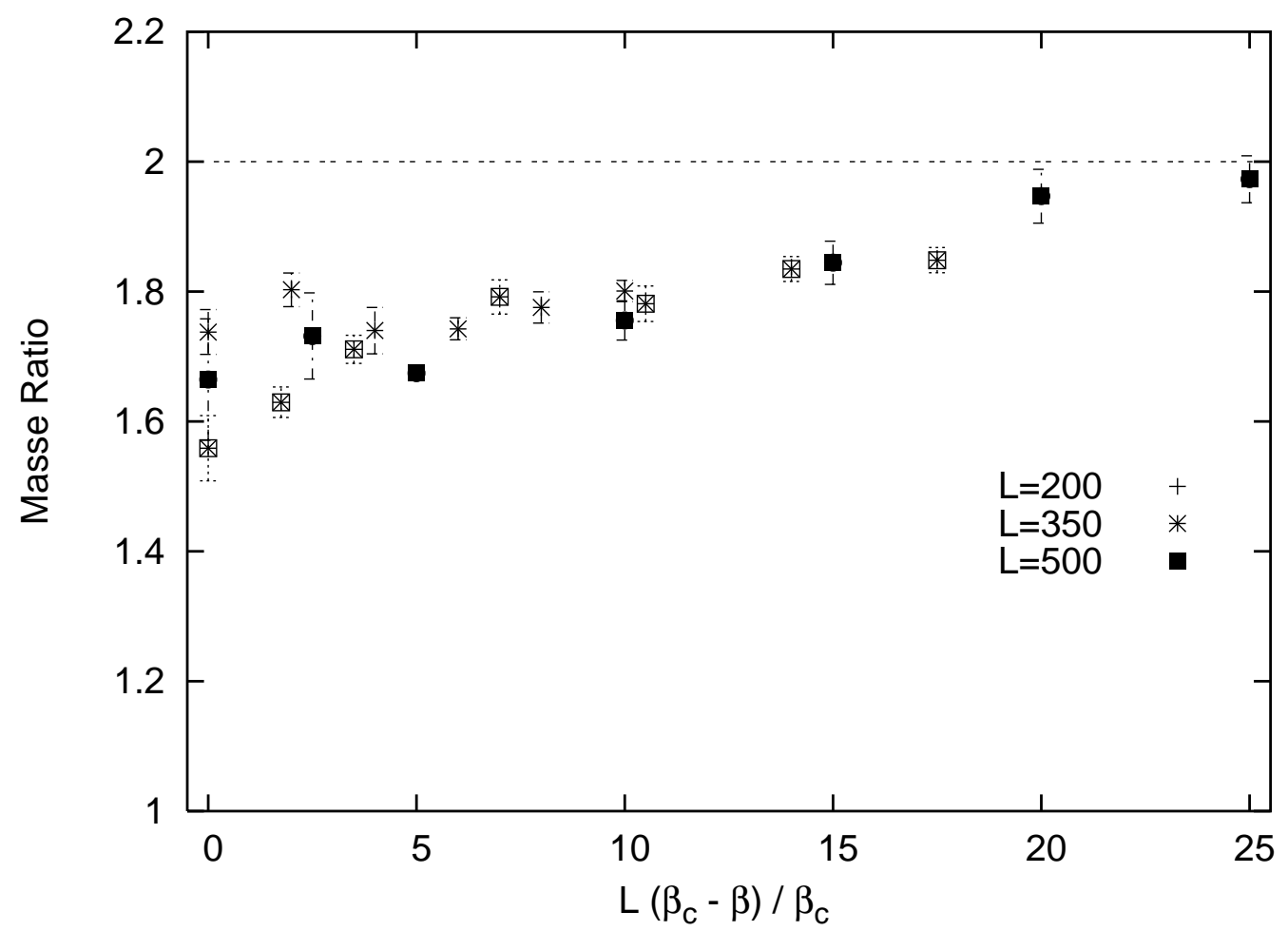

Figure 2: Finite size scaling plot of the mass ratio (see text) versus $L^{1 / \nu}\left(\beta_{c}-\beta\right) / \beta_{c}$ for the $q=3$ Potts model.

length) for the $q=3$ states Potts model, $J_{1} / J_{2}=10$ and $L=500$ is plotted in figure 1 as a function of $\beta<\beta_{c}$. Throughout this paper we used the jack-knife method to estimate statistical errors. We found that statistical errors are smaller than systematic errors. We applied a small $x$ cutoff $x_{0}$ and a large $x$ cutoff $x_{1}$ in fitting the data, because, as expected, close to the boundaries the previous exponential form is not valid. The choice of $x_{0}$ and $x_{1}$ is an important source of systematic errors on the mass $\mu$. For every size $L$ and every $\beta$ we tried all pairs of $x_{0}$ and $x_{1}$ for which the fit was reasonable, i.e. the $\chi^{2}$ of the fit per degree of freedom was $.10 \leq \chi^{2} \leq .80$ (the values of $m(x)$ at different $x$ are not statistically independent). The error bars in figure 1 reflect the dependence of the mass $\mu$ on the different choices of "acceptable" cutoff's (statistical errors are much smaller). Typical values were $5 \leq x_{0} \leq 15$ and $.5 L \leq x_{1} \leq .8 L$ depending on $L$ and $\beta$. We performed a similar analysis of $m_{(2)}(x)$ defined above and measured in the same way the corresponding mass $\mu_{2}$. The ratio $R=\mu_{2} / \mu$ is dimensionless. It is plotted in figure 2 as a function of the scaling variable $L^{1 / \nu}\left(\beta_{c}-\beta\right) / \beta_{c}$ for the $q=3$ states Potts model and $J_{1} / J_{2}=10$. Following the results of previous simulations [10, we assumed that the critical exponent $\nu=1$.

Figure 2 shows that the behavior of $R$ is compatible with the scaling ansatz. For $\beta$ far from $\beta_{c} R=2$, i.e. for large $x, m_{(2)}(x)$ decays twice as fast as $m(x)$. This shows that, far from criticality, the correlation length is self-averaging.

Near the critical point, $R<2$. As this is discussed in [1] this implies the existence of a bound state in the underlying field theory. In the case of the RFIM $R=1$, which means that the bound state completely dominates the asymptotic long distance behavior. In the $q=3$ random Potts model $1<R<2$, which suggests a weaker coupling of the bound state, as we will see.

As this is discussed in [1] if there exist more than one comparable mass scales, as it can be in the case of bound states, we expect a superposition of exponentials governing the long 


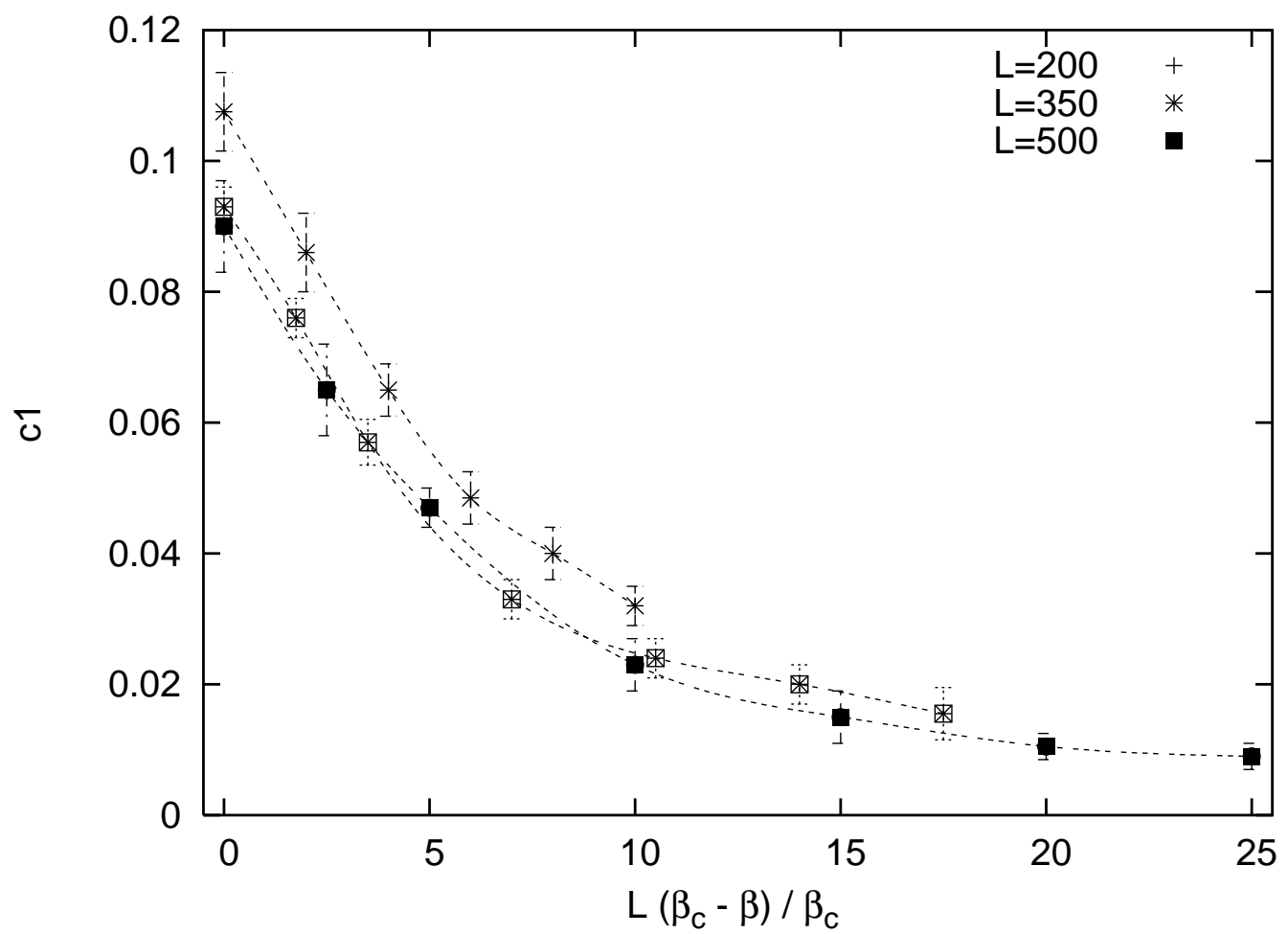

Figure 3: Finite size scaling plot of $c_{1}$ (see text) for the $q=3$ Potts model.

distance behavior. This was the case for the RFIM.

We performed a similar analysis and fitted our data as follows:

$$
m_{(2)}(x)=c_{1} m(x)+c_{2}(m(x))^{2} .
$$

It turned out that this two parameter fit of $m_{2}(x)$ is better than the four parameter fit of eq. (2). If $c_{1} \neq 0$ the first term dominates for large $x\left(m(x)\right.$ decreases much slower than $\left.m(x)^{2}\right)$ and the mass is non self-averaging. As for the RFIM we made the finite size scaling hypothesis that the dimensionless coefficient $c_{1}$ is a function of $L^{1 / \nu}\left(\beta_{c}-\beta\right) / \beta_{c}$. In figure 3 we plot $c_{1}$ versus $L\left(\beta_{c}-\beta\right) / \beta_{c}$ (we took $\nu=1$ ) for different values of $\beta$ and $L=200,350$ and 500 . We see that for $L=500$ and $L=350$ the data fall completely on each other, while for $L=200$ small non leading corrections to scaling seem to be present. For $\beta=\beta_{c}, c_{1}=.10 \pm .02$. We conclude that for the $q=3$ random Potts model the mass is not self-averaging for any finite volume, provided the temperature is close enough to its critical value. This is due to the presence of bound states in the replica field theory, as in the case of the RFIM. The violation of self-averaging is much weaker than for the random field model in three dimensions where we found $c_{1}=1$, i.e the maximum possible violation of self-averaging.

We performed a similar analysis for the Ising model with $J_{1} / J_{2}=5$. In figure 4 we plot $c_{1}$ versus $L\left(\beta_{c}-\beta\right) / \beta_{c}$. At first sight the data seem again compatible with this scaling hypothesis and there is again evidence of violation of self-averaging of the mass, although weaker than in the Potts case $\left(c_{1} \sim .033\right.$ at $\left.\beta_{c}\right)$. A closer look to the data at $\beta=\beta_{c}$, however, leaves open the possibility of a slow crossover to $c_{1}=0$ at $\beta_{c}$ as $L \rightarrow \infty$. (Away from $\beta_{c}$ scaling seems to work fine.) In order to settle this question we simulated a larger lattice with $L=700$ at $\beta_{c}$. If scaling of $c_{1}$ holds, $c_{1}$ should be independent of $L$ at $\beta=\beta_{c}$. We found the following values of $c_{1}$ at $\beta_{c}: .034 \pm .003$ for $L=700, .0355 \pm .004$ for $L=500, .0365 \pm .004$ for $L=350$ and $.0425 \pm .004$ for $L=200$. $c_{1}$ seems to decrease from $L=200$ to $L=350$ and then change very little from $L=350$ to $L=500$ and to $L=700$. It is known[7] that the renormalization group 


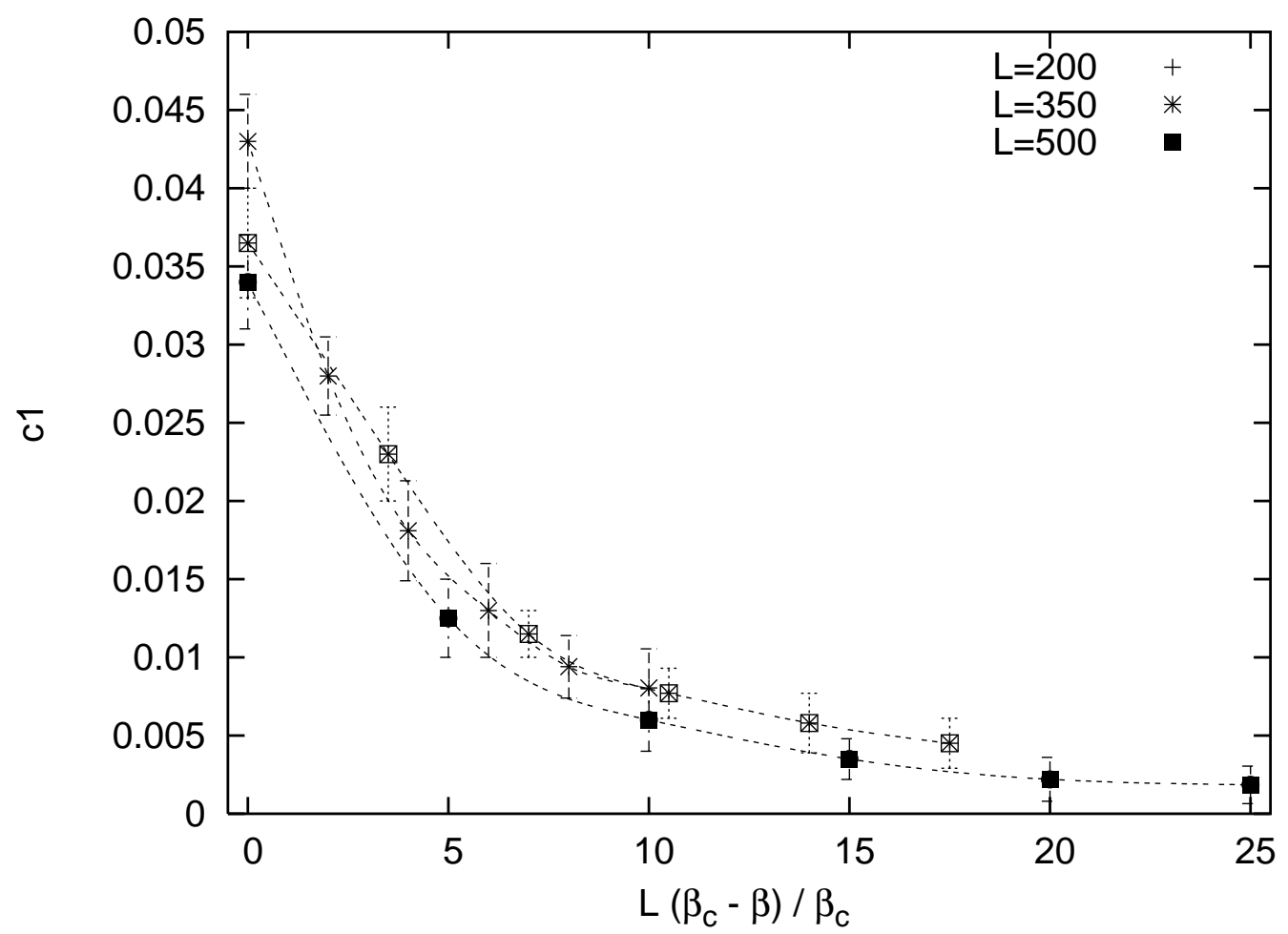

Figure 4: Finite size scaling plot of $c_{1}$ (see text) for the Ising model.

fixed point of the random Ising model flows to the pure Ising fixed point at a logarithmic rate. Our data cannot discriminate between such a behavior or sub-dominant corrections to scaling when the lattice is not large enough.

Finally in order to verify the dependency of the violations of self-averaging of the CL on the disorder, i.e. the ratio $r=J_{1} / J_{2}$, and the number of states of the Potts model $q$, we measured $c_{1}$ for $L=200$ and $\beta=\beta_{c}$ for other values of $q$ and $r$. For $q=8$ and $r=10$ we got $c_{1}=.19 \pm .02$. For $q=2$ (Ising) we got $c_{1}=.0145 \pm .005$ for $r=2$ and $c_{1}=.063 \pm .002$ for $r=10$. We conclude that $c_{1}$, i.e. the violations of self-averaging of the correlation length, increase when the disorder is larger, or when the number $q$ of states of the Potts model increases.

\section{References}

[1] G. Parisi and N. Sourlas, Phys. Rev. Lett. 89, 257204 (2002).

[2] A. B. Harris, J. Phys. C7, 1671 (1974).

[3] A. Aharony and A. B. Harris Phys. Rev. Lett. 77 , 3700; S. Wiseman and E. Domany Phys. Rev. Lett. 81, 22 (1998); H. G. Ballesteros, L. A. Fernández, V. Martin-Mayor, A. Mañoz Sudipe, G. Parisi and J. J. Ruiz-Lorenzo, Phys. Rev. B 58, 2740 (1998).

[4] N. Sourlas, Europhys. Lett. 31007 (1987).

[5] A. W. W. Ludwig, Nucl. Phys. B 285, 97 (1987); ibid. B 330, 639 (1990).

[6] Vl. Dotsenko, M. Picco and P. Pujol, Nucl. Phys. B 455 [FS], 701 (1995).

[7] Vik. Dotsenko and Vl. Dotsenko, Sov. Phys. JETP Lett. 33, 37 (1981).

[8] W. Kinzel and E. Domany, Phys. Rev. B 23, 3421 (1981).

[9] R. H. Swendsen and J. S. Wang, Phys. Rev. Lett. 58, 86 (1987); U. Wolff, Phys. Rev. Lett. 62, 361 (1989).

[10] J. L. Jacobsen, Phys. Rev. E 61 R6060 (2000). 
[11] J. L. Jacobsen and M. Picco, Phys. Rev. E 61 R13 (2000). 